\title{
Revisión sistemática sobre los programas de Entrenamiento Socioemocional para niños y adolescentes de 6 a 18 años publicados entre 2011 y 2015 \\ Systematic Review of Social-Emotional Training Programs For Children and Adolescents
}

\author{
Josefina Rubiales, Daiana Russo, J. Pablo \\ Paneiva y Rocío González \\ Universidad Nacional de Mar del Plata \\ Consejo Nacional de Investigaciones Científicas y \\ Técnicas, Argentina
}

\begin{abstract}
Resumen
En las últimas décadas ha aumentado el número de publicaciones sobre programas de entrenamiento socioemocional y se consideran como factores protectores que facilitan la adaptación de la persona al contexto y favorecen un mejor afrontamiento ante diferentes situaciones de la vida, así como ante situaciones de estrés. El objetivo del presente trabajo fue realizar una revisión sistemática de programas de entrenamiento socioemocional realizados en niños y adolescentes con el fin de seleccionar los más apropiados. El proceso de búsqueda se efectuó a partir de las bases de datos: PsycInfo, MedLine, ERIC, Red de Revistas Científicas de América Latina y El Caribe, Scielo, PUBMED, con palabras claves en inglés y español. Los resultados muestran que, de los 19 artículos que fueron seleccionados por cumplir con los criterios, se identificaron 17 programas de entrenamiento, la mayoría realizados en España y en el período 2011-2015. Los objetivos fueron, en mayor medida, destinados a entrenar la inteligencia emocional, seguido por los entrenamientos mixtos y en menor medida los destinados a entrenar las habilidades sociales. Se identificaron las técnicas empleadas en estos programas, la duración promedio de las intervenciones, la modalidad de aplicación, los administradores y los destinatarios. Por último, del total de los estudios analizados, la mayoría evidencia efectividad del entrenamiento para algunas de las variables analizadas. La evidencia presentada puede ser de utilidad para investigadores y profesionales del área de la salud y la educación que trabajen con niños y adolescentes.
\end{abstract}

Palabras clave: Programas de entrenamiento, Inteligencia emocional, Habilidades sociales, Competencia socioemocional, Niños, Adolescentes

Josefina Rubiales, Daiana Russo, J. Pablo Paneiva y Rocío González; Instituto de Psicología Básica, Aplicada y Tecnología (IPSIBAT). Consejo Nacional de Investigaciones Científicas y Técnicas (CONICET). Facultad de Psicología, Universidad Nacional de Mar del Plata.

La correspondencia en relación con este artículo se dirige a Dra. Josefina Rubiales; Facultad de Psicología, Universidad Nacional de Mar del Plata. Dirección electrónica: josefinarubiales@gmail.com 


\begin{abstract}
:
In recent decades the number of publications on social-emotional training programs has increased, achieving consideration as protective factors that facilitate the adaptation of the individual to their context and allow better coping with different life situations, as well as of stressful situations. The objective of the present study was to perform a systematic review of social-emotional training programs in children and adolescents in order to select the most appropriate ones. The search process was carried out in the following databases: PsycInfo, MedLine, ERIC, Red de Revistas Científicas de América Latina y el Caribe, Scielo, PUBMED, with key words in English and Spanish and certain inclusion criteria. The results showed that of the 19 articles that were selected because they met the criteria, 17 training programs were identified, most of them carried out in Spain during the 2011-2015 period. The objectives of these training programs were to a greater extent, adapted to emotional intelligence, followed to a lesser extent by mixed training. The techniques used in these programs were identified, together with the duration of the interventions, the mode of application, the administrators and the recipients. Finally, of the total studies analyzed, most evidenced the effectiveness of training for some of the variables analyzed. The evidence presented may be useful for researchers and professionals in the area of health and education who work with children and adolescents.
\end{abstract}

Keywords: Training Programs, Emotional Intelligence, Social Skills, Social-Emotional Competence, Children, Adolescents.

En los años noventa, comenzó a cuestionarse la educación tradicional y se valoraron los aspectos socioemocionales en los procesos de aprendizaje en el ámbito de la educación formal (Abarca-Castillo, 2003; Bisquerra-Alzina, 2005; Souza-Barcelar, 2012). Surge así el interés por el desarrollo de programas de educación emocional (Lamas, 2013; Rodríguez \& Montanero, 2017) al concebir a la escuela como una red social protectora que permite nutrir el desarrollo infantil y potenciar el entrenamiento social y afectivo de los estudiantes al generarun clima más positivo para el aprendizaje(Oros, Manucci, \& Richaud de Minzi, 2011).

Bisquerra-Alzina y Álvarez-Fernández (2000) definen la educación emocional como un proceso educativo, continuo, permanente, planificado y desarrollado, a través de programas, con carácter de prevención primaria inespecífica, dirigido a potenciar el desarrollo socioemocional; es decir, el conjunto de conocimientos, capacidades, habilidades y actitudes necesarias para comprender, expresar y regular de forma apropiada los fenómenos socioemocionales como complemento indispensable del desarrollo cognitivo (Pérez-González, 2008). Esta perspectiva implica, entonces, contemplar las emociones y las habilidades sociales como un contenido que puede aprehenderse a través del proceso de interacción (Bisquerra-Alzina, 2003; Brackett \& Caruso, 2007; López-Goñi \& Goñi-Zabalza, 2012). Por lo que, en las últimas décadas, se ha incrementado el número de publicaciones sobre el desarrollo e implementación de programas de entrenamiento socioemocional al entenderlos como procesos educativos, planificados y realizados mediante programas, dirigidos al desarrollo integral de la persona a través del aprendizaje y práctica de aspectos sociales y emocionales (Bisquerra-Alzina, 2003; Pérez-González, 2008).

Esas investigaciones tienen mayor interés en la etapa infantil, en la cual las habilidades socioemocionales se van constituyendo y desarrollando, aunque su estudio resulta complejo debido a la escasez de instrumentos de medida consistentes destinados a evaluar los aspectos socioemocionales en niños (Castillo \& Greco, 2015; Rivers et al., 2012), que dificultan la evaluación y la eficacia de los programas de intervención (Brackett \& Salovey, 2006; Saarni, 2001). 
Sin embargo, es preciso destacar que el desarrollo de programas de entrenamiento socioemocional hace referencia a diversos constructos que podrían ser objeto de dicho entrenamiento; entre ellos se incluyen la Inteligencia Emocional (IE), las Habilidades Sociales (HHSS) y las Competencias Socioemocionales (CSE).

\section{Inteligencia Emocional}

El concepto de IE surge en la década del noventa y desde entonces se han elaborado un gran número de teorías basadas principalmente en dos modelos: el modelo que considera a la IE como rasgo y el modelo de IE entendida como habilidad (Díaz, 2013; Extremera-Pacheco \& Fernández-Berrocal, 2016). El modelo de rasgo o mixto concibe a la IE como una combinación de habilidades emocionales y rasgos de personalidad y la entiende como una característica innata (Valadez, Pérez, \& Beltrán, 2010), dentro de los cuales se destaca el modelo de la IE social de Bar-On (1997) y el modelo de las competencias emocionales de Goleman (1995).

Por su parte, el modelo de habilidad considera a la IE como un conjunto de habilidades cognitivas necesarias para el procesamiento de la información emocional en contextos intra e interpersonales (Zafra, Martos, \& Martos, 2014), en la que se destaca el modelo de Mayer, Caruso y Salovey (1999), que actualmente tiene mayor aceptación en la literatura científica (Fernández-Berrocal \& Extremera-Pacheco, 2004). Estos autores conceptualizan la IE como la capacidad para identificar, evaluar y diferenciar las emociones propias y las de los demás, saber utilizarlas en la toma de decisiones, comprenderlas y regular tanto las emociones positivas como las negativas en uno mismo y en otros (Mayer, Caruso, \& Salovey, 1999). Consideran cuatro dimensiones en la IE ordenadas en forma jerárquica, en función de su conformación psicoevolutiva: percepción, (capacidad de percibir las emociones en sí mismos y en los demás), facilitación (la capacidad de utilizar las emociones para potenciar y dirigir el pensamiento y para dirigir la creatividad y la resolución de problemas), comprensión (la capacidad de comprender la combinación de diversas emociones y la transición emocional, es decir, cómo una emoción se puede transformar en otra, y de analizar las emociones en cada uno de sus componentes) y regulación emocional (la capacidad de gestionar las emociones propias y las de los demás, moderando las emociones negativas y valorando las positivas con el objetivo de lograr una buena y adecuada adaptación) (Luna \& López-Barajas, 2016; Mestré, Guil, Brackett, \& Salovey, 2008; Zeidner, Matthews, Roberts, \& MacCann, 2003).

Este modelo, a diferencia de los modelos mixtos, cuenta con definiciones más precisas, apoyadas por estudios empíricos y prescinde de conceptos próximos a la personalidad. Asimismo, resulta de gran interés para la psicología, porque se concibe a la IE, no como un rasgo o característica interna que resulta heredable e inmodificable, sino como la puesta en práctica de habilidades y comportamientos susceptibles de aprendizaje, desarrolloy entrenamiento (Billings, Downey, Lomas, Lloyd, \& Stough, 2014; Oberst \& Lizeretti, 2004).

La IE ha sido relacionada con numerosas variables criterio. Presenta implicaciones sobre indicadores de ajuste, bienestar, salud, felicidad, personalidad (Mikolajczak et al., 2015; Rey, Extremera, \& Pena, 2016; Vergara, Alonso-Alberca, San-Juan, Aldás, \& Vozmediano, 2015) y se observa específicamente que mayores niveles en esta habilidad se relaciona con una mejor salud tanto física como mental, con un mayor bienestar, un mejor funcionamiento social y rendimiento escolar, menor agresividad, entre otros (Gutiérrez-Cobo, Cabello-González, \& Fernández-Berrocal, 2017). 


\section{Habilidades sociales}

Las HHSS se definen como las conductas o destrezas sociales específicas, requeridas para ejecutar competentemente una tarea de índole interpersonal (Monjas-Casares, 2006). Los autores que abordan el constructo han consensuado algunos elementos comunes para su definición: las HHSS se pueden enseñar, modificar y mejorar mediante los mecanismos básicos del aprendizaje; son recíprocas y dependen de la conducta de las otras personas que se encuentren en el contexto; contienen componentes verbales y no verbales, cognitivos, emocionales y fisiológicos; aumentan el refuerzo social y la satisfacción mutua; y dependen de la situación y sus reglas, y de las personas implicadas y sus características (Gil \& León, 2011; Monjas-Casares, 2006).

Si bien los términos de HHSS y competencia social suelen emplearse como sinónimos, son constructos independientes pero relacionados. Las HHSS tienen un carácter descriptivo y hacen referencia a aquellas conductas necesarias para un buen desempeño social (Morán \& Olaz, 2014; Rodríguez \& Montanero, 2017). Por su parte, el concepto de competencia social no solo incluye la posesión y la utilización de esas habilidades, que implican pensamientos, emociones y conductas para conseguir el éxito en los comportamientos sociales, sino que también implica que los mismos sean valorados como adecuados en el contexto cultural que se encuentren (Gilar-Corbi, Miñano-Pérez, \& Castejón-Costa, 2008; Monjas-Casares, 2006). En este sentido, tanto la competencia social como las HHSS son constructos multidimensionales, siendo considerada la competencia social la superordenada de ambos conceptos (Pichardo, García, Justicia, \& Llanos, 2008).

\section{Competencias socioemocionales}

La delimitación del constructo de CSE es un tema en debate en el que aún no existe acuerdo (Bisquerra-Alzina \& Pérez-Escoda, 2007), de todas maneras, se han propuesto diversas definiciones entre las que se destaca la conceptualización realizada por Saarni (2001). Dicha autora asocia la competencia socioemocional con la demostración de autoeficacia; es decir, con la capacidad y la habilidad de expresar emociones en las transacciones sociales, para lo cual se requiere conocimiento de las propias emociones y la capacidad para regularlas en función de los resultados deseados tomando especial relevancia la importancia del contexto.

Desde esta línea, Bisquerra-Alzina y Pérez-Escoda (2007) definen al concepto de CSE como el conjunto de conocimientos, habilidades y actitudes necesarias para comprender, expresar y regular de forma apropiada los fenómenos emocionales. Se considera que, como toda competencia, incluye capacidades informales y de procedimiento además de las formales. Son pasibles de desarrollo y aprendizaje continuo a través de la experiencia y se inscriben en un contexto determinado.

De esta manera, se observa que la competencia socioemocional es un constructo práctico que pone mayor énfasis en la interacción entre la persona y su ambiente y se vincula, de este modo, al aprendizaje y al desarrollo (Bisquerra-Alzina \& Pérez-Escoda, 2007; Romero, Guajardo, \& Nava, 2017).

\section{Entrenamiento de las habilidades socioemocionales}

Los estudios en esta área de conocimiento han evidenciado que las competencias socioemocionales son útiles como factores protectores que facilitan la adaptación de la persona al contexto y favorecen un 
mejor afrontamiento ante diferentes situaciones de la vida, así como ante situaciones de estrés (GutiérrezCobo, Cabello-González, \& Fernández-Berrocal, 2017; Mikulic, Crespi, \& Radusky, 2015). En el área educativa, se ha evidenciado un beneficio generado por un adecuado aprendizaje de las competencias socioemocionales, porque tienen implicancias en los procesos de aprendizaje, la calidad del desempeño, el éxito académico y la solución de problemas (Bisquerra \& Pérez, 2007; Gómez-Ortiz, Romera, \& OrtegaRuiz, 2017; Zins, Weissberg, Wang, \& Walberg, 2004). En el área de la salud mental, se ha evidenciado que el desarrollo de competencias socioemocionales contribuye al logro de un buen ajuste psicosocial y al bienestar emocional favoreciendo la salud física y mental (Bisquerra-Alzina, 2003; Extremera-Pacheco \& Fernández-Berrocal, 2013; Mayer, Roberts, \& Barsade, 2008; Petrides, Pérez-González, \& Furnham, 2007).

En relación con ello, la orientación educativa actual está concediendo en forma creciente un papel relevante a la formación en competencias socioemocionales de los estudiantes, lo cual fomenta el aprendizaje de estas mediante programas de entrenamiento destinados tanto a la educación infantil, primaria y secundaria, como universitaria (Barrón-Sánchez \& Molero, 2014; Bisquerra-Alzina \& Pérez-Escoda, 2007; Extremera-Pacheco \& Fernández-Berrocal, 2016).

Más allá de los beneficios que los programas de entrenamiento puedan generar en las variables mencionadas, no siempre han sido acompañados con pruebas claras sobre su eficacia (Pérez-González, 2008; Repetto-Talavera, Pena-Garrido, Mudarra, \& Uribarri, 2007) y los estudios sobre el tema aún no aportan evidencias concluyentes. No solo basta con diseñar y aplicar programas educativos que pretendan desarrollar las competencias socioemocionales, sino también es preciso evaluar estas intervenciones, tanto para contar con datos empíricos acerca de su mayor o menor grado de validez como para detectar aquellos aspectos de tales intervenciones que sean susceptibles de mejora (Pérez-González, 2008). De esta manera, el objetivo del presente estudio es realizar una revisión sistemática de los programas de entrenamiento socioemocional existentes en la actualidad al reunir los resultados de investigaciones recientes sobre dichos programas realizados en niños y adolescentes y analizando las técnicas de intervención existentes y sus características.

La revisión sistemática de la evidencia científica es una herramienta metodológica de investigación que ofrece la posibilidad de actualizar e integrar la información disponible de forma eficiente con métodos sistemáticos y explícitos para identificar, seleccionar y valorar las investigaciones relevantes (Meca \& Ausina, 2010; Perestelo-Pérez, 2013). Por lo tanto, esta herramienta podría ser de gran utilidad para el abordaje del estudio de los programas de entrenamiento socioemocional con el objetivo de alcanzar una comprensión del estado actual, lo que permite el desarrollo de transferencias que posibiliten aportar cambios y proponer programas de entrenamiento acordes con las necesidades de la población infanto-juvenil.

\section{Método}

El presente artículo ha seguido el formato propuesto y sugerido para las revisiones sistemáticas por los últimos avances al respecto (Perestelo-Pérez, 2013).

\section{Criterios de selección de los estudios}

Se realizaron búsquedas computarizadas en enero de 2016, en las bases de datos PsycInfo, MedLine, ERIC, Red de Revistas Científicas de América Latina y El Caribe (Redalyc), Scielo, PUBMED, con las 
palabras claves en inglés: training programs - social skills - emotional intelligence - socialemotional skills - socialemotional competencies; y en español: programas de entrenamiento - habilidades sociales - inteligencia emocional - habilidades socioemocionales - competencias socioemocionales.

\section{Criterios de inclusión}

(1) Los trabajos debían haber sido publicados entre 2005 y 2016; (2) debían estar escritos en idioma inglés o español; (3) debían ser diseños con grupo experimental y grupo control y se descartan los diseños $\mathrm{N}=1$, (3) debían estar realizados en población general, no en población clínica; y (4) debían referirse a intervenciones en niños y adolescentes de edades entre 6 y 18 años.

El proceso de búsqueda se basó en la consulta de 1595 referencias. Una vez realizadas las búsquedas en las distintas fuentes de información, dos revisores, de forma independiente, procedieron a preseleccionar las referencias potencialmente relevantes por los títulos y los resúmenes según los criterios de inclusión. Después de revisar títulos y resúmenes, se seleccionaron 26 publicaciones que cumplirían con los criterios de inclusión. Una vez finalizada la fase de preselección, se procedió a repetir la metodología anterior con los artículos completos para seleccionar los artículos que se analizaron y sintetizaron en la revisión. Este procedimiento permitió identificar un total de 19 artículos que cumplieron con los criterios de selección, incluyendo un total de 45 variables analizadas. En total se recogieron datos de 3233 niños y adolescentes de grupo de intervención y 1915 niños y adolescentes de grupo control, con una mortalidad media en torno al $11.18 \%$.

\section{Proceso de codificación de los estudios}

Las características de los estudios fueron codificadas con el fin de analizar las comparaciones tanto a corto como a largo plazo. Se agruparon en tres categorías: variables sustantivas (de intervención, de sujeto y de contexto), variables metodológicas y variables extrínsecas (Meca \& Ausina, 2010).

Las características de intervención codificadas fueron las siguientes: (a)nombre delaintervención;(b) objetivos delaintervención (habilidadessociales, emocionalesy mixtos); (c)técnicas de intervención;(d)soporte(oral, escrito o mixto);(e)duración(en semanas);(f)intensidad (horas semanales); (g) magnitud(horas totales);(h)modo de aplicación (grupal, individual o mixto); e(i) quien lo aplica (docente, profesional, otros).

Las características codificadas de los participantes para las muestras de cada estudio fueron las siguientes: (a) la edad media de la muestra (en años); (b) la desviación estándar de la edad de la muestra poblacional y (c) el género de la muestra (porcentaje de varones).

En cuanto a las características metodológicas, se codificaron las siguientes: (a) $\mathrm{N}$ del grupo experimental; (b) N del grupo control; (c) el criterio de selección de los participantes; (d) el modo de asignación de los participantes a los grupos de intervención (de forma voluntaria o al azar); (e) las medidas pre y pos intervención; (f) la mortalidad experimental (porcentaje de sujetos que abandonan el programa); (g) si realizan o no seguimiento posterior y $(\mathrm{h})$ si incluyen a otra muestra poblacional de estudio.

Las medidas pre y pos intervención (e) fueron reagrupadas de acuerdo con las siguientes categorías: 1) salud mental: consiste en un puntaje total y puntajes individuales para las variables de (a) autoestima y autoeficacia, (b) depresión, (c) ansiedad, (d) síntomas y problemas sociales y emocionales, 
(e) locus de control, (f) estrés y (g) ajuste psicológico; 2) competencias sociales: competencias sociales, resolución de problemas sociales, habilidades sociales, toma de decisiones sociales, prosocial y empatía 3) competencias emocionales: comprensión emocional, reconocimiento emocional, inteligencia emocional, competencia emocional, conocimiento emocional y alfabetización emocional; 4) variables sociales grupales: bienestar socioemocional, integración social, mapa sociocognitivo o sociograma (número de elecciones) y clima institucional; 5) agresividad: conducta agresiva y oposicionista, conducta antisocial, agresividad y bullying; 6) rendimiento académico; 7) consumo de drogas; 8) funcionamiento ejecutivo; y 9) satisfacción en la escuela.

Como variables del contexto solo se codificó el lugar de aplicación (aula o gimnasio). Por último, las características extrínsecas codificadas fueron las siguientes: (a) el año de publicación del trabajo; (b) el país de proveniencia.

La codificación de algunas características requirió la adopción de juicios de decisión complejos. Con objeto de contrastar la adecuación de tales juicios, se realizó un estudio de la fiabilidad del proceso de codificación. Para ello, dos investigadores codificaron de forma independiente una muestra de los estudios (el 26\% del total). El grado de acuerdo alcanzado fue obtenido a partir del índice de Tasa de Acuerdo (Agreement Rate) propuesto por Orwin (1994), el cual en promedio se situó en torno al $90 \%$ en todas las variables codificadas, lo que resulta altamente satisfactorio. Las inconsistencias entre los codificadores se resolvieron por consenso.

\section{Resultados}

Los 19 artículos que fueron seleccionados para la presente revisión se presentan en la tabla 1, en la cual pueden observarse los programas de entrenamiento encontrados, su objetivo y las técnicas de intervención utilizadas. Se encontraron 17 programas de entrenamiento, de los cuales INTEMO y Mindfulness fueron utilizados en dos estudios cada uno.

Los artículos estaban escritos en inglés y español, y procedían de 11 países. Se encontró que en mayor proporción los estudios (47.36\%) fueron realizados en España, y los restantes en diversos países en igual proporción (5.26\%): Suecia, Irán, EE.UU., Bolivia, Nigeria, Canadá, República Libanesa, Portugal, Alemania y Chile. Respecto a las fechas de publicación, el $26.31 \%$ de los artículos fueron publicados en el período 2005-2010 frente al 73.68\% de los artículos publicados en el período 2011-2015. 
Tabla 1

Variables de programas de intervención

\begin{tabular}{|c|c|c|c|c|}
\hline \multirow[b]{2}{*}{ Código } & \multirow[b]{2}{*}{ Autores, año, país } & \multicolumn{3}{|c|}{ Variables del programa } \\
\hline & & Nombre & Objetivo & Técnicas de intervención \\
\hline 1 & $\begin{array}{l}\text { Ambrona, López-Pérez } \\
\text { y Márquez-González, } \\
\text { 2012, España. }\end{array}$ & $\begin{array}{l}\text { Programa Educación } \\
\text { Emocional } \\
\text { Cooperativo } \\
\text { (EDEMCO). }\end{array}$ & $\begin{array}{l}\text { Inteligencia } \\
\text { Emocional }\end{array}$ & $\begin{array}{l}\text { Rompecabezas, en la cual cada niño } \\
\text { tiene un rol dentro del grupo. }\end{array}$ \\
\hline 2 & $\begin{array}{l}\text { Berger, Milicic, } \\
\text { Alcalay y Torretti, } \\
\text { 2014, Chile. }\end{array}$ & $\begin{array}{l}\text { Programa Bienestar } \\
\text { y Aprendizaje } \\
\text { Socioemocional } \\
\text { (BASE) }\end{array}$ & Mixto & $\begin{array}{l}\text { Actividades de juego, tareas que } \\
\text { implican distintas formas de expresión } \\
\text { (escrita, dibujos, dramatizaciones). }\end{array}$ \\
\hline 3 & $\begin{array}{l}\text { Mateu-Martínez, } \\
\text { Piqueras, Jiménez- } \\
\text { Albiar, Espada, } \\
\text { Carballo y Orgilés, } \\
\text { 2013, España. }\end{array}$ & $\begin{array}{l}\text { Programa cognitivo- } \\
\text { conductual para } \\
\text { favorecer las } \\
\text { relaciones y } \\
\text { prevenir el rechazo } \\
\text { social }\end{array}$ & Mixto & $\begin{array}{l}\text { Resolución de problemas, } \\
\text { reestructuración cognitiva, modelado, } \\
\text { autocontrol, entrenamiento en } \\
\text { habilidades sociales, técnicas operantes } \\
\text { para la adquisición y mantenimiento } \\
\text { de conductas, técnicas de control de la } \\
\text { activación (respiración diafragmática y } \\
\text { relajación muscular). }\end{array}$ \\
\hline 4 & $\begin{array}{l}\text { Petermann y Natzke, } \\
\text { 2008, Alemania. }\end{array}$ & $\begin{array}{l}\text { Projet Prima!r' in } \\
\text { Luxembourg }\end{array}$ & Mixto & $\begin{array}{l}\text { Tareas cognitivas-conductuales: } \\
\text { programas de contingencia y juego de } \\
\text { roles. }\end{array}$ \\
\hline 5 & $\begin{array}{l}\text { Raimundo, Marques- } \\
\text { Pinto y Lima, 2013, } \\
\text { Portugal. }\end{array}$ & $\begin{array}{l}\text { Social-emotional } \\
\text { learning (SEL) } \\
\text { Program }\end{array}$ & Mixto & $\begin{array}{l}\text { Instrucción, actividades de narración, } \\
\text { reflexión, lluvia de ideas, modelado, } \\
\text { juegos de rol, realimentación } \\
\text { constructiva, social y auto-refuerzo. }\end{array}$ \\
\hline 6 & $\begin{array}{l}\text { Castillo, Salguero, } \\
\text { Fernández- Berrocal } \\
\text { y Balluerka, 2013, } \\
\text { España. }\end{array}$ & $\begin{array}{l}\text { INTEMO program } \\
\text { (Ligado a SEL) }\end{array}$ & $\begin{array}{l}\text { Inteligencia } \\
\text { Emocional }\end{array}$ & $\begin{array}{l}\text { Actividad de foco emocional: juegos, } \\
\text { juegos de rol, proyectos de arte, foros } \\
\text { de cine y actividades de reflexión. }\end{array}$ \\
\hline 7 & $\begin{array}{l}\text { Cejudo y Latorre, } \\
\text { 2015, España. }\end{array}$ & SPOK (videojuego) & $\begin{array}{l}\text { Inteligencia } \\
\text { Emocional }\end{array}$ & Reforzamiento \\
\hline
\end{tabular}

Continúa $\ldots$ 


\begin{tabular}{|c|c|c|c|c|}
\hline 8 & $\begin{array}{l}\text { Cruz-Colmenero, } \\
\text { Caballero-García y } \\
\text { Ruiz-Tendero, 2013, } \\
\text { España. }\end{array}$ & $\begin{array}{l}\text { Programa de } \\
\text { entrenamiento } \\
\text { en expresión y } \\
\text { reconocimiento de } \\
\text { emociones a través } \\
\text { de la dramatización }\end{array}$ & $\begin{array}{l}\text { Inteligencia } \\
\text { Emocional }\end{array}$ & $\begin{array}{l}\text { Ejercicios dramáticos, instrucciones, } \\
\text { reforzamiento y modelado. }\end{array}$ \\
\hline 9 & $\begin{array}{l}\text { El Hassan y Mouganie, } \\
\text { 2014, República } \\
\text { Libanesa. }\end{array}$ & $\begin{array}{l}\text { The Social } \\
\text { Decision-Making } \\
\text { Skills Curriculum } \\
\text { (SDSC). }\end{array}$ & Mixto & $\begin{array}{l}\text { Entrenamiento en escucha activa } \\
\text { y rol playing, tormenta de ideas } \\
\text { y entrenamiento en solución de } \\
\text { problemas. }\end{array}$ \\
\hline 10 & $\begin{array}{l}\text { Franco-Justo, Fuente- } \\
\text { Arias, y Salvador- } \\
\text { Granados, 2011, } \\
\text { España. }\end{array}$ & $\begin{array}{l}\text { Programa de } \\
\text { meditación, } \\
\text { conciencia plena } \\
\text { (mindfulness) }\end{array}$ & $\begin{array}{l}\text { Inteligencia } \\
\text { Emocional }\end{array}$ & $\begin{array}{l}\text { Mindfulness atendiendo a la } \\
\text { respiración, ejercicio de exploración } \\
\text { corporal. }\end{array}$ \\
\hline 11 & $\begin{array}{l}\text { Hindes, Thorne, } \\
\text { Schwean y McKeough, } \\
\text { 2008, Canadá. }\end{array}$ & $\begin{array}{l}\text { Teen Leadership } \\
\text { Breakthrough } \\
\text { Program (TLB) }\end{array}$ & $\begin{array}{l}\text { Habilidades } \\
\text { Sociales }\end{array}$ & $\begin{array}{l}\text { Actividades experienciales, instrucción } \\
\text { directa y realimentación para enseñar } \\
\text { habilidades de liderazgo. }\end{array}$ \\
\hline 12 & $\begin{array}{l}\text { Pérez-Escoda, Filella, } \\
\text { Alegre y Bisquerra, } \\
\text { 2012, España. }\end{array}$ & $\begin{array}{l}\text { Emotional } \\
\text { Education } \\
\text { Programme for } \\
\text { the Development } \\
\text { of Emotional } \\
\text { Competence in } \\
\text { Primary School } \\
\text { Children. }\end{array}$ & Mixto & $\begin{array}{l}\text { Dinámicas grupales, reflexión, debates } \\
\text { y ejercicio de role-play. }\end{array}$ \\
\hline 13 & $\begin{array}{l}\text { Ruiz-Aranda, Castillo, } \\
\text { Salguero, Cabello, } \\
\text { Fernández-Berrocal } \\
\text { y Balluerka, 2012, } \\
\text { España. }\end{array}$ & $\begin{array}{l}\text { The emotional } \\
\text { intelligence training } \\
\text { (INTEMO) program }\end{array}$ & $\begin{array}{l}\text { Inteligencia } \\
\text { Emocional }\end{array}$ & $\begin{array}{l}\text { Trabajo grupal, role-playing, } \\
\text { actividades artísticas, foros fílmicos y } \\
\text { actividades de reflexión. }\end{array}$ \\
\hline 14 & $\begin{array}{l}\text { Soriano-Ayala y Justo, } \\
\text { 2010, España. }\end{array}$ & $\begin{array}{l}\text { Instruction } \\
\text { in Emotional } \\
\text { Intelligence Skills } \\
\text { on Locus of Control }\end{array}$ & $\begin{array}{l}\text { Inteligencia } \\
\text { Emocional }\end{array}$ & Mindfulness. \\
\hline
\end{tabular}

Continúa... 


\begin{tabular}{|c|c|c|c|c|}
\hline 15 & $\begin{array}{l}\text { Umaru y Umma, 2015, } \\
\text { Nigeria. }\end{array}$ & $\begin{array}{l}\text { Instruction } \\
\text { in Emotional } \\
\text { Intelligence Skills } \\
\text { on Locus of Control }\end{array}$ & $\begin{array}{l}\text { Inteligencia } \\
\text { Emocional }\end{array}$ & Instrucción. \\
\hline 16 & $\begin{array}{l}\text { Pichardo, García, } \\
\text { Justicia y Llanos, } \\
\text { 2008, Bolivia. }\end{array}$ & $\begin{array}{l}\text { ¿Qué divertido es } \\
\text { aprender habilidades } \\
\text { sociales! }\end{array}$ & $\begin{array}{l}\text { Habilidades } \\
\text { Sociales }\end{array}$ & Sin descripción. \\
\hline 17 & $\begin{array}{l}\text { DiPerna, Lei, } \\
\text { Bellinger y Cheng, } \\
\text { 2015, Estados Unidos. }\end{array}$ & $\begin{array}{l}\text { Social Skills } \\
\text { Improvement } \\
\text { System Classwide } \\
\text { Intervention } \\
\text { Program (SSIS- } \\
\text { CIP) }\end{array}$ & $\begin{array}{l}\text { Habilidades } \\
\text { Sociales }\end{array}$ & $\begin{array}{l}\text { Describir, modelado, role-play, } \\
\text { práctica, monitoreo del progreso y } \\
\text { generalización. }\end{array}$ \\
\hline 18 & $\begin{array}{l}\text { Eslami, } \\
\text { Ghofranipour,Bonab, } \\
\text { Zadeh, Shokravi y } \\
\text { Tabatabaie, 2015, Irán. }\end{array}$ & $\begin{array}{l}\text { Evaluation of } \\
\text { a school based } \\
\text { educational } \\
\text { program to prevent } \\
\text { adolescents' } \\
\text { problem behavior }\end{array}$ & $\begin{array}{l}\text { Habilidades } \\
\text { Sociales }\end{array}$ & $\begin{array}{l}\text { Discusiones interactivas, revisión } \\
\text { de reuniones anteriores, role-play y } \\
\text { práctica. }\end{array}$ \\
\hline 19 & $\begin{array}{l}\text { Kimber, Sandell y } \\
\text { Bremberg, 2008, } \\
\text { Suecia. }\end{array}$ & SET Program & Mixto & $\begin{array}{l}\text { Modelado, role-play, práctica, } \\
\text { interacción con los padres. }\end{array}$ \\
\hline
\end{tabular}

La tabla 2 presenta las principales características descriptivas de las variables codificadas en los estudios.

En la tabla 2, puede observarse que, en cuanto al objetivo de los programas de entrenamiento, el más frecuente fue la Inteligencia Emocional (42.1\%), seguido por los entrenamientos mixtos (36.84\%) y siendo el menos frecuente las Habilidades Sociales (21.05\%). En cuanto a los soportes de intervención, el $64.7 \%$ utilizaron recursos escritos y orales, el $17.65 \%$ orales y el $5.89 \%$ escritos. En lo que respecta al modo de aplicación, el $84.21 \%$ de los programas optaron por hacerlo de forma grupal, el $10.53 \%$ de forma individual y grupal y el $5.26 \%$ de forma individual y por parejas. Los administradores más frecuentes fueron los docentes $(47.06 \%)$ y los psicólogos entrenados (47.06\%). En cuanto al lugar de entrenamiento, la mayoría optó por aplicarlo en el aula (88.25\%). 
Tabla 2

Principales variables cuantitativas codificadas en los estudios

\begin{tabular}{|c|c|c|c|}
\hline Variables & Categorías & Frecuencia & $\%$ \\
\hline \multirow[t]{3}{*}{ Objetivo $(k=19)$} & 1. Inteligencia Emocional & 8 & 42.1 \\
\hline & 2. Habilidades Sociales & 4 & 21.05 \\
\hline & 3. Mixto & 7 & 36.84 \\
\hline \multirow[t]{5}{*}{ Soporte $(k=17)$} & 1. Oral & 3 & 17.65 \\
\hline & 2. Escrito & 1 & 5.89 \\
\hline & 3. Mixto & 11 & 64.7 \\
\hline & 4. Videojuego & 1 & 5.88 \\
\hline & 5. Videograbación y Oral & 1 & 5.88 \\
\hline \multirow[t]{3}{*}{ Modo de aplicación $(k=19)$} & 1. Grupal & 16 & 84.21 \\
\hline & 2. Individual + Grupal & 2 & 10.53 \\
\hline & 3. Individual + Parejas & 1 & 5.26 \\
\hline \multirow[t]{3}{*}{ Administrador $(k=17)$} & 1. Docente & 8 & 47.06 \\
\hline & 2. Psicólogo entrenado & 8 & 47.06 \\
\hline & 3. Mixto & 1 & 5.88 \\
\hline \multirow[t]{3}{*}{ Lugar de entrenamiento $(k=17)$} & 1. Aula & 15 & 88.25 \\
\hline & 2. Aula de informática & 1 & 5.87 \\
\hline & 3. Sala de Expresión dramática / Gimnasio & 1 & 5.87 \\
\hline \multirow{2}{*}{$\begin{array}{l}\text { Realizan seguimiento posterior }(k= \\
\text { 18) }\end{array}$} & 1. $\mathrm{Si}$ & 7 & 36.84 \\
\hline & 2. No & 12 & 63.16 \\
\hline \multirow[t]{3}{*}{ Selección de los sujetos } & 1. Al azar & 3 & 11.76 \\
\hline & 2. Por conveniencia & 12 & 70.59 \\
\hline & 3. Aceptación de la escuela & 2 & 17.65 \\
\hline \multirow{4}{*}{$\begin{array}{l}\text { Asignación de los sujetos a los } \\
\text { grupos }(k=17)\end{array}$} & 1. Al azar & 10 & 58.82 \\
\hline & 2. Intencional & 4 & 23.53 \\
\hline & 3. Al azar controlando sexo & 2 & 11.76 \\
\hline & 4. Al azar controlando ubicación & 1 & 5.89 \\
\hline
\end{tabular}

Nota. $k=$ número de estudios que informaron la variable analizada. 
El método de selección de los participantes fue por conveniencia en el 70.59\% de los estudios, al azar en el $11.76 \%$, y el restante $17.65 \%$ fueron instituciones educativas que aceptaron participar en el estudio, habiendo sido invitadas junto con otras instituciones que no aceptaron. La asignación de estos participantes a cada uno de los grupos fue en un 76.47\% al azar (de los cuales 2 estudios controlaron género y un estudio la ubicación) y un $23.53 \%$ intencional.

En la tabla 3 se presentan los datos de las variables de los participantes y de las variables metodológicas para el grupo con intervención -GI- y el grupo control de los estudios codificados -GC-.

Tabla 3

Variables de los participantes y variables metodológicas para ambos grupos de los estudios codificados

\begin{tabular}{|c|c|c|c|c|c|}
\hline \multirow[b]{2}{*}{ Cód. } & \multicolumn{3}{|c|}{ Variables de los participantes } & \multicolumn{2}{|c|}{ Variables metodológicas } \\
\hline & $M$ Edad & $D E$ Edad & $\begin{array}{c}\text { Género } \\
\text { (\% varones) }\end{array}$ & $N$ GI & $N$ GC \\
\hline 1 & 6.23 & 0.43 & 48.33 & 30 & 30 \\
\hline 2 & 9.15 & $\mathrm{~S} / \mathrm{D}$ & 51.5 & 437 & 234 \\
\hline 3 & 9.75 & 0.96 & $\mathrm{~S} / \mathrm{D}$ & 67 & 27 \\
\hline 4 & 7 & $\mathrm{~S} / \mathrm{D}$ & 50.8 & 46 & 42 \\
\hline 5 & 9.31 & 0.56 & 55.03 & 213 & 105 \\
\hline 6 & 13.83 & 1.5 & 45.59 & 361 & 229 \\
\hline 7 & $17 / 19$ & $\mathrm{~S} / \mathrm{D}$ & 48.9 & 92 & 50 \\
\hline 8 & $10 / 11$ & $\mathrm{~S} / \mathrm{D}$ & 46.93 & 23 & 22 \\
\hline 9 & $7 / 9$ & $\mathrm{~S} / \mathrm{D}$ & $\mathrm{S} / \mathrm{D}$ & 80 & 51 \\
\hline 10 & 17.06 & 2.44 & 29 & 42 & 42 \\
\hline 11 & 16.1 & $14.2 / 18.3$ & 45.45 & 41 & 36 \\
\hline 12 & $6 / 12$ & $\mathrm{~S} / \mathrm{D}$ & 51.3 & 223 & 200 \\
\hline 13 & 13 & 0.87 & 47.4 & 322 & 157 \\
\hline 14 & 16.45 & 0.78 & 39 & 25 & 24 \\
\hline 15 & Secundarios & $\mathrm{S} / \mathrm{D}$ & 50 & 20 & 20 \\
\hline 16 & $6 / 9$ & $\mathrm{~S} / \mathrm{D}$ & 53 & 76 & 74 \\
\hline 17 & 7.355 & 0.38 & $\mathrm{~S} / \mathrm{D}$ & 228 & 204 \\
\hline 18 & 15 & 0.72 & 100 & 49 & 57 \\
\hline 19 & $1^{\circ}$ a $7^{\circ}$ grado & $\mathrm{S} / \mathrm{D}$ & $\mathrm{S} / \mathrm{D}$ & 352 & 110 \\
\hline
\end{tabular}

Nota. $\mathrm{S} / \mathrm{D}=$ Sin datos; $M=$ media; $D E=$ desviación estándar; $N=$ número; $\mathrm{GI}=$ Grupo con Intervención; $\mathrm{GC}=$ Grupo Control. 
REVISIÓN SISTEMÁTICA SOBRE LOS PROGRAMAS DE ENTRENAMIENTO SOCIOEOMOCIONAL

Respecto los datos de los participantes, la edad media fue de 11.69 años y el 50.82\% de los participantes eran varones. En cuanto a las variables metodológicas, el tamaño de los grupos ha sido variable, con $N$ mínimo de 20 y máximo de 437 niños, con una media de 143 niños por grupo de intervención.

Respecto a otras variables metodológicas no incluidas en la tabla, se menciona que la duración promedio de la intervención fue de 11.71 semanas; el mínimo corresponde a 2 semanas y el máximo 24. La intensidad promedio de la intervención fue de 93 minutos semanales. El mínimo era de 30 minutos y el máximo de 360 minutos, mientras que la magnitud promedio de la intervención fue de 14.57 horas, con un mínimo de 6 horas y un máximo de 25 horas. Del total de los estudios, 5 presentaron datos sobre la mortalidad experimental de los participantes con un $11.18 \%$.

En la tabla 4 pueden observarse los estadísticos descriptivos, media y $D E$, para ambos grupos en el pre y postest.

Tabla 4

Estadísticos descriptivos discriminados por grupos en el pre y postest y resumen de los resultados para las variables codificadas en los estudios

\begin{tabular}{|c|c|c|c|c|c|c|}
\hline \multirow[t]{2}{*}{ Cód. } & \multirow[t]{2}{*}{ Categoría } & \multicolumn{2}{|c|}{ Grupo control } & \multicolumn{2}{|c|}{ Grupo intervención } & \multirow{2}{*}{ Resumen } \\
\hline & & Pre & Post & Pre & Post & \\
\hline 1 & Competencia emocional & $7.82(2.47)^{\mathrm{a}}$ & $7.85(1.55)$ & $7.64(1.31)$ & $9.32(1.87)$ & $\mathrm{GI} \neq \mathrm{GC}$ \\
\hline \multirow[t]{3}{*}{2} & Variables sociales grupales & $53.72(9.34)$ & $54.94(9.61)$ & $53.18(9.86)$ & $53.72(10.47)$ & $\mathrm{GI} \neq \mathrm{GC}$ \\
\hline & $\begin{array}{l}\text { Salud Mental. Autoestima y } \\
\text { Autoeficacia. }\end{array}$ & $39.67(7.43)$ & $39.05(8.37)$ & $35.21(8.99)$ & $39.30(8.52)$ & \\
\hline & Rendimiento académico & $6.10(.45)$ & $5.94(.49)$ & $5.36(1.83)$ & $5.76(.56)$ & \\
\hline \multirow[t]{4}{*}{3} & Integración social & $7.56(8.57)$ & $7.67(8.15)$ & $8.75(7.20)$ & $9.58(7.51)$ & $\mathrm{GI} \neq \mathrm{GC}$ \\
\hline & $\begin{array}{l}\text { Inteligencia social } \\
\text { emocional }\end{array}$ & $24.99(4.09)$ & $20.71(3.56)$ & $24.21(4.69)$ & $25.03(4.61)$ & \\
\hline & Salud mental. Depresión & $9.70(4.57)$ & $11.33(4.31)$ & $12.28(7.62)$ & $11.60(7.73)$ & \\
\hline & Salud mental. Ansiedad & $34.78(12.72)$ & $37.93(10.45)$ & $29.69(15.00)$ & $27.09(14.48)$ & \\
\hline \multirow[t]{4}{*}{4} & $\begin{array}{l}\text { Salud mental. Síntomas } \\
\text { y problemas sociales y } \\
\text { emocionales }\end{array}$ & $8.74(7.13)$ & $8.84(8.15)$ & $7.95(8.00)$ & $8.74(7.13)$ & $\mathrm{GI} \neq \mathrm{GC}$ \\
\hline & Competencia social & $2.54(.69)$ & $2.63(.78)$ & $3.16(.78)$ & $2.54(.69)$ & \\
\hline & Competencia emocional & $10.29(3.08)$ & $10.63(3.72)$ & $14.89(3.62)$ & $10.29(3.08)$ & \\
\hline & Agresividad & $4.76(4.11)$ & $5.76(5.39)$ & $5.28(5.71)$ & $4.76(4.11)$ & \\
\hline
\end{tabular}

Continúa... 


\begin{tabular}{|c|c|c|c|c|c|c|}
\hline \multirow[t]{5}{*}{5} & Competencia emocional & $\mathrm{S} / \mathrm{D}$ & $\mathrm{S} / \mathrm{D}$ & $\mathrm{S} / \mathrm{D}$ & $\mathrm{S} / \mathrm{D}$ & \\
\hline & Competencia social & $4.06(.81)$ & $3.90(.83)$ & $3.63(.83)$ & $3.78(.83)$ & $\begin{array}{l}\text { (comp. } \\
\text { Social y }\end{array}$ \\
\hline & Salud mental. Ansiedad & $\mathrm{S} / \mathrm{D}$ & $\mathrm{S} / \mathrm{D}$ & $\mathrm{S} / \mathrm{D}$ & $\mathrm{S} / \mathrm{D}$ & $\begin{array}{c}\text { emocional); } \\
G \mathrm{~J}=\mathrm{GC}\end{array}$ \\
\hline & Agresividad & $1.52(.59)$ & $1.43(.65)$ & $1.62(.69)$ & $1.46(.60)$ & (en Salud \\
\hline & Problemas sociales & $1.06(.15)$ & $1.12(.23)$ & $1.20(.25)$ & $1.19(.31)$ & $\begin{array}{c}\text { mental y } \\
\text { agresividad) }\end{array}$ \\
\hline \multirow[t]{2}{*}{6} & Agresividad & $2.63(.77)$ & $2.67(.75)$ & $2.55(.78)$ & $2.47(.74)$ & $\mathrm{GI} \neq \mathrm{GC}$ \\
\hline & Competencia social & $3.06(.61)$ & $3.12(.62)$ & $3.05(.64)$ & $3.10(.62)$ & \\
\hline 7 & Competencia emocional & $95.28(9.41)$ & $94.10(8.26)$ & $96.98(10.31)$ & $100.05(6.44)$ & $\mathrm{GI} \neq \mathrm{GC}$ \\
\hline 8 & Competencia emocional & $5.97(1.14)$ & $8.50(5.32)$ & $6.80(1.26)$ & $7.61(1.43)$ & $\mathrm{GI} \neq \mathrm{GC}$ \\
\hline \multirow[t]{2}{*}{9} & Competencia social & $\mathrm{S} / \mathrm{D}$ & $71.77(12.80)$ & $\mathrm{S} / \mathrm{D}$ & $72.91(11.56)$ & $\mathrm{GI} \neq \mathrm{GC}$ \\
\hline & Competencia emocional & $\mathrm{S} / \mathrm{D}$ & $\mathrm{S} / \mathrm{D}$ & $\mathrm{S} / \mathrm{D}$ & $\mathrm{S} / \mathrm{D}$ & \\
\hline 10 & $\begin{array}{l}\text { Salud mental. Autoestima y } \\
\text { autoeficacia }\end{array}$ & $1.83(.62)$ & $1.85(.65)$ & $1.80(.54)$ & $2.46(.50)$ & $\mathrm{GI} \neq \mathrm{GC}$ \\
\hline \multirow[t]{2}{*}{11} & Competencia emocional & $86.60(13.40)$ & $96.30(10.80)$ & $91.20(12.90)$ & $\begin{array}{l}106.20 \\
(10.30)\end{array}$ & $\mathrm{GI} \neq \mathrm{GC}$ \\
\hline & $\begin{array}{l}\text { Salud mental. Autoestima y } \\
\text { Autoeficacia }\end{array}$ & $98.10(9.50)$ & $98.90(11.20)$ & $95.60(15.20)$ & $\begin{array}{l}106.40 \\
(15.10)\end{array}$ & \\
\hline \multirow[t]{3}{*}{12} & Competencia emocional & $6.24(1.14)$ & $6.24(1.19)$ & $6.12(.81)$ & $6.49(1.10)$ & $\mathrm{GI} \neq \mathrm{GC}(6$ \\
\hline & Salud mental. Estrés & $16.51(4.88)$ & $16.61(4.94)$ & $16.68(4.74)$ & $14.39(3.55)$ & $\begin{array}{l}\text { a } 8 \text { anos }) ; \\
\mathrm{GI}=\mathrm{GC}(8\end{array}$ \\
\hline & Variables sociales grupales & $25.54(3.93)$ & $25.87(3.81)$ & $25.00(4.75)$ & $27.73(3.56)$ & $\begin{array}{c}\text { a } 12 \text { años) } \\
\text {; GI } \neq \text { GC } \\
\text { (Variables } \\
\text { sociales) }\end{array}$ \\
\hline \multirow[t]{2}{*}{13} & Salud mental & $.79(.29)$ & $.75(.32)$ & $.77(.33)$ & $.73(.30)$ & $\mathrm{GI} \neq \mathrm{GC}$ \\
\hline & Competencia emocional & $2.32(.64)$ & $2.32(.68)$ & $2.24(.72)$ & $2.29(.69)$ & \\
\hline \multirow[t]{2}{*}{14} & $\begin{array}{l}\text { Salud mental. Autoestima y } \\
\text { Autoeficacia. }\end{array}$ & $27.32(8.37)$ & $28.71(9.76)$ & $26.81(7.44)$ & $36.43(8.71)$ & $\mathrm{GI} \neq \mathrm{GC}$ \\
\hline & Competencia emocional. & $19.43(5.35)$ & $20.79(6.32)$ & $19.75(6.99)$ & $26.20(6.61)$ & \\
\hline \multirow[t]{2}{*}{15} & $\begin{array}{l}\text { Salud mental. Locus de } \\
\text { control }\end{array}$ & $\mathrm{S} / \mathrm{D}$ & $\mathrm{S} / \mathrm{D}$ & $24.80(2.80)$ & $46.35(3.14)$ & $\mathrm{GI} \neq \mathrm{GC}$ \\
\hline & Rendimiento académico & $\mathrm{S} / \mathrm{D}$ & $\mathrm{S} / \mathrm{D}$ & $26.45(2.29)$ & $46.35(3.14)$ & \\
\hline
\end{tabular}

Continúa... 
REVISIÓN SISTEMÁTICA SOBRE LOS PROGRAMAS DE ENTRENAMIENTO SOCIOEOMOCIONAL

\begin{tabular}{|c|c|c|c|c|c|c|}
\hline \multirow[t]{2}{*}{16} & Competencia social & $11.80(\mathrm{~S} / \mathrm{D})$ & 4.25 (S/D) & $23.94(\mathrm{~S} / \mathrm{D})$ & $40.96(\mathrm{~S} / \mathrm{D})$ & \multirow{2}{*}{$\begin{array}{c}\mathrm{GI} \neq \mathrm{GC} \\
\text { (Comp. } \\
\text { social); } \\
\mathrm{GI}=\mathrm{GC} \\
\text { (Conducta } \\
\text { antisocial) }\end{array}$} \\
\hline & $\begin{array}{l}\text { Agresividad. conducta } \\
\text { antisocial }\end{array}$ & $17.36(\mathrm{~S} / \mathrm{D})$ & 33.81 (S/D) & $39.65(\mathrm{~S} / \mathrm{D})$ & $36.63(\mathrm{~S} / \mathrm{D})$ & \\
\hline 17 & Competencia social & $1.48(.57)$ & $1.50(.59)$ & $1.51(.53)$ & $1.61(.52)$ & $\mathrm{GI} \neq \mathrm{GC}$ \\
\hline \multirow[t]{2}{*}{18} & Competencia social & $34.90(9.30)$ & $35.00(8.80)$ & $34.80(10.10)$ & $39.50(9.90)$ & \multirow[t]{2}{*}{$\mathrm{GI} \neq \mathrm{GC}$} \\
\hline & Consumo de drogas & $3.65(.29)$ & $3.67(.28)$ & $3.64(.32)$ & $3.56(.30)$ & \\
\hline \multirow[t]{6}{*}{19} & $\begin{array}{l}\text { Salud mental. Autoestima y } \\
\text { autoeficacia }\end{array}$ & $1.00(.38)$ & $.92(.47)$ & $.89(.45)$ & $0.92(.45)$ & \multirow{6}{*}{$\begin{array}{c}\mathrm{GI}=\mathrm{GC} \\
(\text { Comp. } \\
\text { social) } ; \mathrm{GI} \\
\neq \mathrm{GC}(\text { Salud } \\
\text { mental })\end{array}$} \\
\hline & $\begin{array}{l}\text { Salud mental. Síntomas y } \\
\text { problemas de salud mental }\end{array}$ & $.38(0.30)$ & $.43(0.36)$ & $.46(0.34)$ & $.45(.33)$ & \\
\hline & $\begin{array}{l}\text { Salud mental. Autoestima y } \\
\text { Autoeficacia }\end{array}$ & $2.23(.44)$ & $2.25(.44)$ & $2.08(.45)$ & $2.09(.46)$ & \\
\hline & Competencia social & $2.07(.44)$ & $2.05(.43)$ & $2.00(.44)$ & $2.02(.42)$ & \\
\hline & Satisfacción en la escuela & $4.38(.65)$ & $4.20(.89)$ & $4.28(.84)$ & $4.22(.86)$ & \\
\hline & Agresividad. Bullying & $1.18(.40)$ & $1.32(.72)$ & $1.23(.48)$ & $1.20(.44)$ & \\
\hline
\end{tabular}

Nota. $\mathrm{S} / \mathrm{D}=$ Sin datos; GI = Grupo con Intervención; GC = Grupo Control.

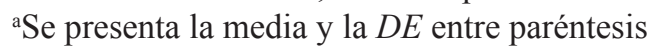

En el estudio 1, el grupo que recibió la intervención mejoró significativamente en todas las variables medidas de competencia emocional (reconocimiento emocional, comprensión emocional simple y comprensión mixta), mientras que no se observaron cambios en el grupo control (Ambrona, LópezPérez, \& Márquez-González, 2012). En el estudio 2 se encontraron diferencias significativas en las variables analizadas y se hallaron mejoras en el grupo con intervención.

En el estudio 3, se evidenciaron mejorías en el grupo con intervención en comparación con el grupo control en el postest en las puntuaciones de la escala de ansiedad e inteligencia social-emocional. Asimismo, en el grupo con intervención, se observa una mejoría entre pretest y postest a partir de un descenso significativo en las variables de ansiedad y depresión y un incremento significativo en integración social e inteligencia social emocional, que incluía la evaluación de autoconciencia, aprovechamiento emocional (automotivación), empatía y habilidad social, manteniéndose estables los índices de rechazos y elecciones (Mateu-Martínez et al., 2013). Además, en el estudio 4 se encontró una reducción en los problemas sociales y emocionales y en agresividad, así como un aumento en las competencias sociales y emocionales para los niños evaluados.

En el estudio 5 se observaron mejorías en algunas variables y desempeños semejantes entre ambos grupos en otras variables. En las variables competencia social y emocional hubo mejorías en el grupo con intervención; sin embargo, en las variables de ansiedad, agresividad y problemas sociales no se observaron diferencias entre los grupos con y sin intervención. 
En el estudio 6 se encontraron mejorías significativas en agresividad y disminuyó en el grupo con intervención luego del tratamiento y mejorías, aunque menores, en la variable de competencia social. En el estudio 7, se observan diferencias entre ambos grupos en la puntuación global de competencia emocional, con mejorías en el grupo con intervención, específicamente en la inteligencia emocional, en percepción emocional, en facilitación emocional y en regulación emocional (Cejudo \& Latorre, 2015). En el estudio 8 se encontró que el programa de entrenamiento permite mejorar el desempeño en la variable de competencia emocional (expresión y reconocimiento de las emociones) y se evidenciaron diferencias entre ambos grupos.

En el estudio 9 se encontraron diferencias entre el pre y postest en el grupo con intervención en la variable competencia emocional y social. En el estudio 10, se encontraron diferencias entre el grupo con intervención y control en el postest en la variable salud mental (compuesta por tres factores: capacidad de afrontamiento, operatividad y realización en el quehacer), autoconcepto y autoestima, y en empatía y realización social. Asimismo, se demostró que el programa produjo un incremento y mejora de las variables en el GI al comparar sus puntuaciones antes y después de la intervención, aspecto que no ocurrió al analizar los datos pretest y postest del grupo control (Franco-Justo, Fuente-Arias, \& Salvador-Granados, 2011). En el estudio 11, se encontraron diferencias significativas en competencia emocional y en las variables de salud mental, autoestima y autoeficacia (Hindes, Thorne, Schwean, \& McKeough, 2008). Además, se encontraron diferencias en el estudio 12 en relación con variables sociales grupales en todas las edades de la muestra poblacional. Sin embargo, en las variables de competencia emocional y salud mental en los niños de 8 a 12 años, no se observaron mejoras en el grupo con intervención respecto al grupo control (Pérez-Escoda, Filella, Alegre, \& Bisquerra-Alzina, 2012).

El estudio 13 sugiere que el programa de inteligencia emocional, creado para desarrollar habilidades para percibir, facilitar, comprender y controlar las emociones, puede ser efectivo para promover la salud mental porque se han evidenciado diferencias pre y post intervención. En el estudio 14, se evidenció un incremento significativo en los valores de autoestima y en competencia emocional en el postest entre el grupo con y sin intervención (Soriano-Ayala \& Justo, 2010).

En el estudio 15, se encontró una mejoría de los niños del grupo con intervención con respecto a la variable locus de control y en el rendimiento académico al comparar las evaluaciones pre y pos intervención. Los resultados revelan que el entrenamiento en inteligencia emocional tuvo efecto positivo sobre el rendimiento académico y el locus de control de los estudiantes del grupo con intervención. No se describieron los resultados del grupo control. En el estudio 16, los niños que participaron en el programa, después de la aplicación, presentaron mejor desempeño en competencia social que el grupo control. Se observó también una disminución en el comportamiento antisocial, aunque las diferencias no son significativas entre la evaluación pre y posintervención del GI. También, se encontraron diferencias significativas en los estudios 17 (en competencia social), 18 (en competencia social y consumo de drogas) y 19 (en salud mental, autoeficacia y agresividad). Se evidenciaron diferencias pre y pos intervención. Los estudios, que han realizado seguimiento fueron el 1, 4, 11, 13, 18 y 19, indicaron que las mejorías continuaron un tiempo después de la intervención, a excepción del estudio 19, que al momento de la publicación no contaba con los resultados del seguimiento a largo plazo. 


\section{Discusión}

Como se ha observado, el desarrollo de programas de entrenamiento socioemocional tiene como propósito general mejorar la calidad de vida y el bienestar tomando diversos indicadores. Sin embargo, no siempre han sido acompañados con pruebas claras sobre su eficacia y las evidencias que aportan no son aún concluyentes (Pérez-González, 2008; Repetto-Talavera et al., 2007). De esta manera, contar con una revisión sistemática de los programas de entrenamiento socioemocional realizados en niños y adolescentes existentes en la actualidad, muestra el estado actual dando lugar a un posible desarrollo de transferencias que posibiliten aportar cambios y proponer programas de entrenamientos acordes con las necesidades de la población infanto-juvenil (Bisquerra-Alzina \& Pérez-Escoda, 2007; Repetto-Talavera et al., 2007).

Los resultados del presente estudio muestran que, de los 19 artículos que fueron seleccionados por cumplir con los criterios, se identificaron 17 programas de entrenamiento, la mayoría realizados en España y en el período 2011-2015, de los cuales solo dos fueron replicados o utilizados en dos estudios cada uno. Los objetivos fueron en mayor medida destinados a entrenar la IE, seguido por los entrenamientos mixtos y en menor medida los destinados a entrenar las HHSS. Entre las técnicas empleadas en estos programas, se identifican rompecabezas, juegos, resolución de problemas, reestructuración cognitiva, modelado, autocontrol, entrenamiento en habilidades sociales, técnicas de control de la activación (respiración y relajación), programas de contingencias, juego de roles, instrucción, actividades de narración, reflexión, lluvia de ideas, realimentación, reforzamiento, mindfulness, actividades artísticas e intervención con padres. La duración promedio de las intervenciones fue de 11.71 semanas, con una intensidad promedio de 93 minutos semanales, siendo la magnitud promedio de 14.57 horas. Estos datos sobre la duración no son fácilmente comparables entre los estudios, ya que los tiempos mínimos y máximos son disímiles. Del total de los estudios, solo 5 presentaron datos sobre la mortalidad experimental de los participantes, que corresponde a $11.18 \%$.

Asimismo, se observa que la mayor proporción de estos estudios (84.21\%) optaron por un modo de aplicación grupal en el espacio de aula. Los administradores más frecuentes fueron docentes (47.06\%) y psicólogos entrenados (47.06\%), los destinatarios fueron niños con una media de edad de 11.69 años y el $50.82 \%$ de los participantes eran varones, seleccionados en mayor medida por muestreo por conveniencia y asignando al azar a los participantes a cada uno de los grupos.

El total de los estudios analizados han tomado como indicadores para analizar la efectividad de los programas las siguientes categorías de variables: salud mental, competencias sociales, competencias emocionales, variables sociales grupales, agresividad, rendimiento académico, consumo de drogas, funcionamiento ejecutivo y satisfacción en la escuela. Las variables más estudiadas han sido competencia emocional y social, salud mental, agresividad y variables sociales grupales.

La totalidad de los estudios analizados, evidencia mejoría con los entrenamientos para algunas de las variables analizadas. Respecto a la variable competencia emocional, seis de los diez estudios evidenciaron efectividad en los programas. En la variable agresividad, los cinco estudios que la analizaron informaron que los entrenamientos fueron efectivos para disminuirla. En cuanto a la efectividad de los programas para mejorar las variables vinculadas con la salud mental, la autoestima y la autoeficacia se 
han visto favorecidas en cuatro estudios; en uno de ellos (estudio 12) el nivel de estrés disminuyó luego de la intervención para la ansiedad un programa fue altamente efectivo y otro no fue efectivo y para la depresión un único estudio no evidenció efectividad.

Respecto a las variables sociales grupales, un estudio demostró ser efectivo y otro no mostró efectividad del entrenamiento. En cuanto a los estudios que analizaron la competencia social, siete de los ocho estudios, evidenciaron efectividad en los programas.

Aquellos programas, que mostraron diferencias significativas luego de las intervenciones, trabajaron en su mayoría como objetivo del entrenamiento la inteligencia emocional (42.1\%). Las técnicas más utilizadas han sido las siguientes: resolución de problemas, reestructuración cognitiva, modelado, autocontrol, reforzamiento positivo social, técnicas de control de la activación (respiración diafragmática y relajación muscular progresiva), mindfulness, instrucción directa y realimentación.

En cuanto a su duración, los cinco programas de mayor efectividad fueron de menor cantidad de horas. Asimismo, utilizan, en mayor medida, grupos con menor cantidad de participantes, que han sido aplicados mayormente a adolescentes y no se encuentran diferencias respecto al género.

En lo que respecta al tipo de soporte del programa, no se evidencian diferencias entre los entrenamientos. En cuanto al modo de aplicación, los resultados sugieren una tendencia a que los estudios de entrenamiento con mayor efectividad fueron aplicados por profesionales de la Psicología.

También, se identificaron vacíos que posiblemente restringen la generalización de los resultados: la falta de unificación en los criterios de clasificación de los componentes de los programas de entrenamiento socioemocional y el poco consenso en los modelos teóricos de partida, la no utilización de instrumentos de evaluación específicos, sensibles y relacionados con el constructo a ser entrenado los cuales permitan identificar los cambios producidos por las intervenciones (porque se han tomado solo variables externas para mostrar la efectividad de los programas), la falta no solo de estudio en nuestro contexto local sino también de replicación de los estudios ya realizados, el empleo de diseños de estudio que no permiten demostrar causalidad y la falta de descripción de algunos datos en los estudios realizados.

Entre las limitaciones del presente estudio, es importante mencionar que no se han incluido aquellos estudios no publicados en revistas científicas o tesis de grado y posgrado que trabajan en la temática y que no se han podido realizar análisis estadísticos de metaanálisis para comparar entre las investigaciones, debido a que no todos los estudios publicaron la totalidad de sus resultados.

A pesar de las limitantes, es relevante conocer los programas de intervención con variables medidas de modo sistematizado de manera tal que puedan ser replicados y que sus resultados puedan ser contrastados, porque el conocer las características que resultan más efectivas al momento de implementar un programa de entrenamiento socioemocional en los contextos educativos podría aportar conocimientos al diseño de futuros abordajes. De esta manera, los hallazgos encontrados en la presente revisión contribuyen a enriquecer el cuerpo de conocimientos de la Psicología y al diseño de programas de entrenamiento socioemocional. Se considera particularmente el rol que tienen en el desarrollo escolar de niños y adolescentes. 


\section{Referencias $^{1}$}

Abarca-Castillo, M. (2003). La educación emocional en la Educación Primaria: Curriculo y práctica (Tesis de doctorado inédita). Universidad de Barcelona, España. Recuperado de http://www.tdx.cat/handle/10803/2349

*Ambrona, T., López-Pérez, B., \& Márquez-González, M. (2012). Eficacia de un programa de educación emocional breve para incrementar la competencia emocional de niños de educación primaria. Revista Española de Orientación y Psicopedagogía, 23(1), 39-49. doi: 10.5944/reop.vol.23.num.1.2012.11392

Bar-On, R. (1997). The Emotional Quotient Inventory (EQ-i): A test of Emotional Intelligence. Toronto: MultiHealth Systems.

Barrón-Sánchez, M. C., \& Molero, D. (2014). Estudio sobre inteligencia emocional y afectos en escolares de educación primaria. Revista Electrónica de Investigación y Docencia, 12, 7-18. Recuperado de http:// revistaselectronicas.ujaen.es/index.php/reid/article/viewFile/1197/1503

*Berger, C., Milicic, N., Alcalay, L., \& Torretti, A. (2014). Programa para el bienestar y aprendizaje socioemocional en estudiantes de tercero y cuarto grado: descripción y evaluación de impacto. Revista Latinoamericana de Psicología, 46(3), 169-177. doi: 10.1016/S0120-0534(14)70020-2

Bisquerra-Alzina, R. (2003). Educación emocional y competencias básicas para la vida. Revista de Investigación Educativa (RIE), 21(1), 7-43. Recuperado de http://revistas.um.es/rie/article/view/99071/94661

Bisquerra-Alzina, R. (2005). La educación emocional en la formación del profesorado. Revista Interuniversitaria de Formación del Profesorado, (54), 95-114. Recuperado de https://dialnet.unirioja.es/servlet/ articulo? codigo $=2126758$

Bisquerra-Alzina, R., \& Álvarez-Fernández, M. (2000). Educación Emocional y Bienestar. Madrid: Praxis.

Bisquerra-Alzina, R., \& Pérez-Escoda, N. (2007). Las competencias emocionales. Educación XXI: Revista de la Facultad de Educación, 10, 61-82. doi: 10.5944/educxx1.1.10.297

Billings, C. E., Downey, L. A., Lomas, J. E., Lloyd, J., \& Stough, C. (2014). Emotional Intelligence and scholastic achievement in pre-adolescent children. Personality and Individual Differences, 65, 14-18. https://doi. org/10.1016/j.paid.2014.01.017

Brackett, M. A., \& Caruso, D. R. (2007). Emotionally Literacy for Educators. Cary, NC: SEL media.

Brackett, M. A., \& Salovey, P. (2006). Measuring emotional intelligence with the Mayer-Salovery-Caruso Emotional Intelligence Test (MSCEIT). Psicothema, 18(Suplemento), 34-41.

*Castillo, R., Salguero, J. M., Fernández-Berrocal, P., \& Balluerka, N. (2013). Effects of an emotional intelligence intervention on aggression and empathy among adolescents. Journal of Adolescence, 36(5), 883-892. doi: 10.1016/j.adolescence.2013.07.001

Castillo, K., \& Greco, C. (2015). Inteligencia emocional: un estudio exploratorio en escolares argentinos de contextos rurales. Revista de Psicología, 23(2), 116-132. doi: 10.5354/0719-0581.2014.36152

*Cejudo, J., \& Latorre, S. (2015). Effects of the Spock videogame on improving emotional intelligence in adolescents. Electronic Journal of Research in Educational Psychology, 13(2), 319-342. doi: 10.14204/ ejrep.36.15060

*Cruz-Colmenero, V., Caballero-García, P., \& Ruiz-Tendero, G. (2013). La dramatización como recurso didáctico para el desarrollo emocional. Un estudio en la etapa de educación primaria. Revista de Investigación Educativa, 31(2), 393-410. doi: 10.6018/rie.31.2.164501

${ }^{1}$ Las referencias marcadas con un asterisco $(*)$, indican los estudios incluidos en la revisión sistemática. 
de Souza-Barcelar, L. (2012). Competencias Emocionales y Resolución de Conflictos Interpersonales en el Aula (Tesis de doctorado inédita). Universidad Autónoma de Barcelona, España. Recuperado de http://www. eumed.net/tesis-doctorales/2012/lsb/indice.htm

Díaz,J.J.M.(2013).Reseñateóricadelainteligenciaemocional:modeloseinstrumentosdemedición.RevistaCientífica, 17, 10-32. Recuperado de http://revistas.udistrital.edu.co/ojs/index.php/revcie/article/view/4505/6254

*DiPerna, J. C., Lei, P., Bellinger, J., \& Cheng, W. (2015). Efficacy of the Social Skills Improvement System Classwide Intervention Program (SSIS-CIP) primary version. School Psychology Quarterly, 30(1), 123141. doi: $10.1037 / \mathrm{spq} 0000079$

*El Hassan, K., \& Mouganie, Z. (2014). Implementation of the Social Decision-Making Skills Curriculum on primary students (Grades 1-3) in Lebanon. School Psychology International, 35(2), 167-175. doi: $10.1177 / 0143034312469758$

*Eslami, A. A., Ghofranipour, F., Bonab, B. G., Zadeh, D. S., Shokravi, F. A., \& Tabatabaie, M. G. (2015). Evaluation of a school-based educational program to prevent adolescents' problem behaviors. Journal of Education and Health Promotion, 4(1), 30. doi: 10.4103/2277-9531.154127

Extremera-Pacheco, N., \& Fernández-Berrocal, P. (2016). Inteligencia Emocional y Educación: Psicología. España: Editorial Grupo 5.

Extremera-Pacheco, N., \& Fernández-Berrocal, P. (2013). Inteligencia emocional en adolescentes. Padres y maestros. Publicación de la Facultad de Ciencias Humanas y Sociales, 352, 34-39. Recuperado de https:// revistas.upcomillas.es/index.php/padresymaestros/article/viewFile/1170/993

*Franco-Justo, C., Fuente-Arias, M. D. L., \& Salvador-Granados, M. (2011). Impacto de un programa de entrenamiento en conciencia plena (mindfulness) en las medidas del crecimiento y la autorrealización personal. Psicothema, 23(1), 58-65. Recuperado de http://www.psicothema.com/PDF/3850.pdf

Fernández-Berrocal, P., \& Extremera-Pacheco, N. (2004). El uso de las medidas de habilidad en el ámbito de la inteligencia emocional: Ventajas e inconvenientes con respecto a las medidas de auto-informe. Boletín de Psicología, (80), 59-78. Recuperado de https://www.uv.es/seoane/boletin/previos/N80-3.pdf

Gil, F., \& León, J. (2011). Habilidades Sociales: Teoría, Investigación e Intervención. Madrid: Síntesis.

Gilar-Corbi, R., Miñano-Pérez, P., \& Castejón-Costa, J. L. (2008). Inteligencia emocional y empatía: su influencia en la competencia social en Educación Secundaria Obligatoria. SUMMA Psicológica UST, 5(1), 21-32.

Goleman, D. (1995). Emotional Inteligence. New York: Bantam Books.

Gómez-Ortiz, O., Romera, E. M., \& Ortega-Ruiz, R. (2017). La competencia para gestionar las emociones y la vida social y su relación con el fenómeno del acoso y la convivencia escolar. Revista Interuniversitaria de Formación del Profesorado, 88(31.1), 27-38.

Gutiérrez-Cobo, M. J., Cabello-González, R., \& Fernández-Berrocal, P. (2017). Programas para mejorar la inteligencia emocional desde el modelo de Mayer y Salovey: sus beneficios en el ámbito psicoeducativo. III Congreso Internacional de Inteligencia Emocional y Bienestar. Zaragoza, España.

*Hindes, Y. L., Thorne, K. J., Schwean, V. L., \& McKeough, A. M. (2008). Promoting intrapersonal qualities in adolescents evaluation of rapport's teen leadership breakthrough program. Canadian Journal of School Psychology, 23(2), 206-222. doi: 10.1177/0829573508327307

*Kimber, B., Sandell, R., \& Bremberg, S. (2008). Social and emotional training in Swedish classrooms for the promotion of mental health: results from an effectiveness study in Sweden. Health Promotion International, 23(2), 134-143. doi: 10.1093/heapro/dam046 
REVISIÓN SISTEMÁTICA SOBRE LOS PROGRAMAS DE ENTRENAMIENTO SOCIOEOMOCIONAL

Lamas,M.C.(2013).Educaciónemocional contribución delaescuelaalasaludmental infantil.RevistaIberoamericana de Educación, 62/63, 1-10. Recuperado de http://www.rieoei.org/deloslectores/5966Lamas.pdf

López-Goñi, I., \& Goñi-Zabalza, J. M. (2012). La competencia emocional en los currícula de formación inicial de los docentes. Un estudio comparativo. Revista de Educación, 357, 1-18. doi: 10-4438/1988-592X-RE-2010-357-069

Luna, N. C., \& López-Barajas, D. M. (2016). Inteligencia emocional percibida, disposición al optimismopesimismo, satisfacción vital y personalidad de docentes en su formación inicial. Revista de Investigación Educativa, 34(1), 241-258. doi: http://dx.doi.org/10.6018/rie.34.1.220701

Mayer, J. D., Caruso, D. R., \& Salovey, P. (1999). Emotional intelligence meets traditional standards for an intelligence. Intelligence, 27(4), 267-298. doi: http://dx.doi.org/10.1016/S0160-2896(99)00016-1

Mayer, J. D., Roberts, R. D., \& Barsade, S. G. (2008). Human abilities: Emotional intelligence. Annual Review of Psychology, 59, 507-536. doi: 10.1146/annurev.psych.59.103006.093646,

*Mateu-Martínez, O., Piqueras, J. A., Jiménez-Albiar, M., Espada, J. P., Carballo, J. L., \& Orgilés, M. (2013). Eficacia de un programa de prevención cognitivo-conductual breve del rechazo social en niños. Terapia Psicológica, 31(2), 187-195. doi: 10.4067/S0718-48082013000200005

Meca, J., \& Ausina, J. (2010). Revisiones sistemáticas y meta-análisis: Herramientas para la práctica profesional. Papeles del Psicólogo, 31(1), 7-17. Recuperado de http://www.um.es/metaanalysis/pdf/5029.pdf

Mestré, J. M., Guil, R., Brackett, M., \& Salovey, P. (2008). Inteligencia emocional: definición, evaluación y aplicaciones desde el modelo de habilidades de Mayer y Salovey. En F. Palmero, F. Martínez Sánchez, \& J. A. Huertas Martínez (Comps.), Motivación y Emoción, (pp. 407-438). España: McGraw-Hill.

Mikolajczak, M., Avalosse, H., Vancorenland, E., Verniest, R., Callens, M., van Broeck, N., .., Mierop, A. (2015). A nationally representative study of emotional competence and health. Emotion, 15(5), 653-667. doi: 10.1037/ emo0000034

Mikulic, I. M., Crespi, M., \& Radusky, P. (2015). Construcción y validación del inventario de competencias socioemocionales para adultos (ICSE). Interdisciplinaria. Revista de Psicología y Ciencias Afines, 32(2). Recuperado de http://www.redalyc.org/pdf/180/18043528007.pdf

Monjas-Casares, M. (2006). Programa de Enseñanza de Habilidades de Interacción Social (PEHIS). Madrid: Cepe.

Morán, V. E., \& Olaz, F. O. (2014). Instrumentos de evaluación de habilidades sociales en América Latina: un análisis bibliométrico. Revista de Psicología, 23(1), 93-105. doi: 10.5354/0719-0581.2014.32877

Orwin, R. G. (1994). Evaluating coding decisions. En H. M. Cooper \& L. V. Hedges (Dirs.), The Handbook of Research Sintesis (pp. 134-172). Nueva York: Sage.

Oberst, U., \& Lizeretti, N. P. (2004). Inteligencia emocional en psicología clínica y en psicoterapia. Revista de Psicoterapia, 60(4), 5-22. Recuperado de http://oberst.es/documentos/OberstLizeretti.pdf

Oros, L. B., Manucci, V., \& Richaud de Minzi, M. C. (2011). Desarrollo de emociones positivas en la niñez. Lineamientos para la intervención escolar. Educación y Educadores, 14(3), 493-509. Recuperado de http:// www.scielo.org.co/pdf/eded/v14n3/v14n3a04

*Pérez-Escoda, N., Filella, G., Alegre, A., \& Bisquerra-Alzina, R. (2012). Developing the emotional competence of teachers and pupils in school contexts. Electronic Journal of Research in Educational Psychology, 10(3), 1183-1208. Recuperado de: http://www.investigacion-psicopedagogica.com/revista/articulos/28/english/ Art $28 \quad 756 . p d f$ 
Pérez-González, J. C. (2008). Propuesta para la evaluación de programas de educación socioemocional. Electronic Journal of Research in Educational Psychology, 6(15), 523-546. Recuperado de http://www.investigacionpsicopedagogica.org/revista/articulos/15/espannol/Art 15 246.pdf

Perestelo-Pérez, L. (2013). Standards on how to develop and report systematic reviews in Psychology and Health. International Journal of Clinical and Health Psychology, 13(1), 49-57. Recuperado de http://aepc.es/ijchp/ articulos pdf/ijchp-432.pdf

*Petermann, F., \& Natzke, H. (2008). Preliminary results of a comprehensive approach to prevent antisocial behaviour in preschool and primary school pupils in Luxembourg. School Psychology International, 29(5), 606-626. doi: $10.1177 / 0143034308099204$

Petrides, K.V., Pérez-González, J.C., \& Furnham,A.(2007). On the criterion and incremental validity of trait emotional intelligence. Cognition and Emotion, 21(1), 26-55. Doi: http://dx.doi.org/10.1080/02699930601038912

*Pichardo, M. C., García, T., Justicia, F., \& Llanos, C. (2008). Efectos de un programa de intervención para la mejora de la competencia social en niños de educación primaria en Bolivia. International Journal of Psychology and Psychological Therapy, 8(3), 441-452. Recuperado de http://www.ijpsy.com/volumen8/ num3/216/efectos-de-un-programa-de-intervencin-para-ES.pdf

*Raimundo, R., Marques Pinto, A., \& Lima, M. L. (2013). The effects of a social-emotional learning program on elementary school children: the role of pupils'characteristics. Psychology in the Schools, 50(2), 165-180. doi: $10.1002 /$ pits.21667

Repetto-Talavera, E., Pena-Garrido, M., Mudarra, J., \& Uribarri, M. (2007). Orientación de las competencias socioemocionales de los alumnos de Educación Secundaria en contextos multiculturales. Electronic Journal of Research in Educational Psychology, 5(11), 159-178. Recuperado de http://www.investigacionpsicopedagogica.org/revista/articulos/11/espannol/Art_11_170.pdf

Rey, L., Extremera, N. \& Pena, M. (2016). Emotional competence relating to perceived stress and burnout in Spanish teachers: a mediator model. PeerJ, 4, 1-14. Doi: 10.7717/peerj.2087

Rivers, S. E., Brackett, M. A., Reyes, M. R., Mayer, J. D., Caruso, D. R., \& Salovey, P. (2012). Measuring emotional intelligence in early adolescence with the MSCEIT-YV psychometric properties and relationship with academic performance and psychosocial functioning. Journal of Psychoeducational Assessment, 30(4), 344-366.

*Ruiz-Aranda, D., Castillo, R., Salguero, J. M., Cabello, R., Fernández-Berrocal, P., \& Balluerka, N. (2012). Shortand midterm effects of emotional intelligence training on adolescent mental health. Journal of Adolescent Health, 51(5), 462-467. doi: http://dx.doi.org/10.1016/j.jadohealth.2012.02.003

Rodríguez, S., \& Montanero, M. (2017). Estudio del entrenamiento en habilidades sociales de un grupo de $2^{\circ}$ de la ESO. Tarbiya, revista de Investigación e Innovación Educativa, (33), 77-90.

Romero, N. A. R., Guajardo, J. G., \& Nava, J. M. F. (2017). Competencias socioemocionales como predictoras de conductas prosociales y clima escolar positivo en adolescentes. Revista interuniversitaria de formación del profesorado, (88), 77-90.

Saarni, C. (2001). Emotional competence: a developmental perspective. En R. Bar-On, \& J. D. A. Parker (Eds.), The Handbook of Emotional Intelligence (pp. 68-91). San Francisco: Jossey-Bass.

*Soriano-Ayala, E., \& Justo, C. (2010). Mejora de la autoestima y de la competencia emocional en adolescentes inmigrantes sudamericanos residentes en España a través de un programa psicoeducativo de Mindfulness (conciencia plena). Revista de Investigación Educativa, 28(2), 297-312. Recuperado de http://revistas. um.es/rie/article/view/99351/121601 
*Umaru, Y., \& Umma, A. (2015). Effect of Instruction in Emotional Intelligence Skills on Locus of Control and Academic Self-Efficacy among Junior Secondary School Students in Niger State, Nigeria. Journal of Education and Practice, 6(18), 164-169. Recuperado de http://files.eric.ed.gov/fulltext/EJ1079778.pdf

Valadez, M. D., Pérez, L., \& Beltrán, J. (2010). La inteligencia emocional de los adolescentes talentosos. Faisca, 15(17), 2-17. Recuperado de http://revistas.ucm.es/index.php/FAIS/article/view/FAIS1010110002A/7640

Vergara, A. I., Alonso-Alberca, N., San-Juan, C., Aldás, J., \& Vozmediano, L. (2015). Be water: Direct and indirect relations between perceived emotional intelligence and subjective well-being. Australian Journal of Psychology, 67, 47-54. doi: 10.1111/ajpy.12065

Zafra, E. L., Martos, M. P., \& Martos, P. B. (2014). EQI-Versión corta (EQI-C). Adaptación y validación al español del EQ-i en universitarios. Boletín de Psicología, 110, 21-36. Recuperado de http://www.uv.es/seoane/ boletin/previos/N110-2.pdf

Zeidner, M., Matthews, G., Roberts, R. D., \& MacCann, C. (2003). Development of emotional intelligence: Towards a multi-level investment model. Human Development, 46(2-3), 69-96. doi:10.1159/000068580

Zins, J. E., Weissberg, R. P., Wang, M. C., \& Walberg, H. J. (2004). Building Academic Auccess on Social and Emotional Learning. New York: Teachers College Press. 


\section{Sobre las autoras y autor:}

Josefina Rubiales es Doctora en Psicología, Magíster en Neuropsicología y Licenciada en Psicología, Investigadora del CONICET-IPSIBAT-UNMDP (Argentina), Profesora en la Licenciatura y en el Doctorado de la Facultad de Psicología de la Universidad de Mar del Plata (Argentina) e integrante del grupo de investigación "Comportamiento Humano, Genética y Ambiente". Trabaja en la línea de investigación sobre toma de decisiones en niños con diagnóstico de TDAH. Cuenta con publicaciones y presentaciones en congresos nacionales e internacionales vinculadas a la temática y cursos y jornadas sobre neurodiversidad y trastornos del neurodesarrollo.

Daiana Russo es Licenciada en Psicología, becaria doctoral del Consejo Nacional de Investigaciones Científicas y Técnicas (CONICET). Cursó la Maestría en Neuropsicología Aplicada (Instituto Universitario del Hospital Italiano de Buenos Aires). Es integrante del grupo de investigación "Comportamiento Humano, Genética y Ambiente" (CONICETIPSIBAT-UNMDP) y responsable de la línea de investigación "Evaluación e intervención en habilidades de solución de problemas interpersonales en niños con diagnóstico de Trastorno por Déficit de Atención e Hiperactividad". Cuenta con publicaciones y presentaciones en congresos nacionales e internacionales vinculadas a la temática.

Juan Pablo Paneiva Pompa es Licenciado en Psicología por la Universidad Nacional de Mar del Plata y se encuentra terminando sus estudios de Especialista en psicoterapia cognitiva (UNMDP - Aiglé). Becario de investigación de la UNMDP. Su tema de investigación corresponde al clima de aula. Forma parte del Grupo de investigación "Comportamiento humano, genética y ambiente" (perteneciente al IPSIBAT: UNMDP-CONICET), cuya línea de investigación se centra en el estudio de la neurodiversidad, especialmente el TDAH. Brinda charlas y talleres para diversos miembros de la comunidad. Labora como docente de la asignatura "Biología Humana" (UNMDP, Facultad de Psicología) y "Psicología Evolutiva" (EUT-CEDiER).

Rocío González es Licenciada en Psicología, Especialista en Psicoterapia Cognitiva, Profesor Adjunta en la Facultad de Psicología y Psicopedagogía de la Universidad del Salvador, Buenos Aires, Argentina. Becaria doctoral CONICET en el Centro Interdisciplinario de Investigaciones en Psicología Matemática y Experimental (CIIPME), Buenos Aires, Argentina. 